\title{
Vaginal Discharge Assessment form for Utilization by Primary Health Care Workers in a Community Setting
}

\author{
Ilankoon IMPS ${ }^{1 *}$, Goonewardena $\mathrm{CSE}^{2}$, Fernandopulle $\mathrm{RC}^{3}$ and Perera PPR \\ ${ }^{1}$ Department of Allied Health Sciences, University of Sri Jayewardenepura, Sri Lanka \\ ${ }^{2}$ Department of Community Medicine, University of Sri Jayewardenepura, Sri Lanka \\ ${ }^{3}$ Department of Obstetrics and Gynaecology, University of Sri Jayawardenapura, Sri Lanka \\ ${ }^{4}$ Department of Biochemistry, University of Sri Jayewardenepura, Sri Lanka
}

Submission: March 06, 2018 ; Published: May 01, 2018

*Corresponding author: IMPS Ilankoon, Department of Allied Health Sciences, Faculty of Medical Sciences, University of Sri Jayewardenepura, Gangodawila, Nugegoda, Sri Lanka, Tel: +94774986623; Email: prasanthi@sjp.ac.lk

\begin{abstract}
Objective: to develop an assessment form for abnormal vaginal discharge to be used by family health care workers/ Public Health Midwives (PHMs) in a community setting especially in developing countries where access to a Gynaecologist may be limited.

Design: Development of the vaginal discharge assessment form was done by the literature review and Delphi technique. Face and content validity were assessed by an expert panel.

Setting: The need assessment was done by focus group discussions and community survey among women living in socially marginalised communities living in Colombo District, Sri Lanka.

Measurements and findings: A vaginal discharge assessment form was developed and validated through content and face validity. The form is a guide for family health care workers (PHMs) working in the community to assess the severity of the symptoms and decide on the actions to be taken for women complaining of abnormal vaginal discharge.

Key conclusion and implications for practice: Family health care workers who engage in community health promotion activities can use the developed vaginal discharge assessment form to take detail history from a woman complaining on vaginal discharge and decide whether to refer the woman to necessary care providers or whether the woman is having normal physiological vaginal discharge. The developed form can be utilised in order to effectively care for women at community level and reduces unnecessary consultations and health cost. Furthermore it would help to refer them promptly to a gynaecologist if a pathological vaginal discharge is suspected.
\end{abstract}

Keywords: Screening tool; Family health care workers; Vaginal complaints; Health promotion; Women's health.

Abbreviations: PHMs: Public Health Midwives; WHO: World Health Organization.

\section{Introduction}

Vaginal discharge is one of the most common gynaecological symptoms affecting women and a very common cause for seeking medical attention [1]. The most common causes for most vaginal symptoms are one of three types of vulvovaginitis: bacterial vaginosis, Candida vulvovaginitis and trichomoniasis $[1,2]$. A chronic discharge must always raise the suspicion of malignancy, and the relevant cytological smears should therefore be performed [1].

Immediate treatment of pathological vaginal discharge could prevent the development of complications or further infection transmission [3]. Therefore, The World Health Organization
(WHO) recommends the syndromic management of vaginal discharge, which is treating for some or all of the five common reproductive tract infections: Chlamydia trachomatis infection, gonorrhea, and trichomoniasis, bacterial vaginosis, and candidiasis, which can cause abnormal vaginal discharge [4]. The unavailability of laboratory support for use of microscopy, cultures and serology, which are further expensive, in many resource-poor countries, have led to development of the syndromic approach [3].

The Colombo city is a relatively large, dense and socially heterogeneous, and is also the major commercial and administrative centres of Sri Lanka [5] which has slum and 
shanty type houses and are occupied by a significant number of low income urban dwellers [5]. There are social issues such as drug addiction, domestic violence, marital instability, and child abuse in these poor urban settlements [5]. The women living in these communities are at risk of different sexual behaviours and at risk of developing sexually transmitted infections and other reproductive tract infections.

According to National STD/AIDS Control Programme, the most commonly found sexually transmitted infections (STIs) in Sri Lanka are genital herpes, genital warts, non-gonococcal infections, syphilis, gonorrhoea, chlamydial infection and trichomoniasis [6]. The highest number of HIV cases reported in 2016 as a total of 249 which might be a fraction of HIV infected people in the country due to stigma and discrimination towards HIV which hinders seeking testing services [6]. Early identification of the signs and symptoms of reproductive tract infections will lead to prevent unnecessary sufferings, health cost and subfertility due to delay in proper treatment.

In the nature of hiding symptoms related to the reproductive and sexual health, women are at risk of developing associated complications and sufferings. Implementation of Primary Health Care Services in Sri Lankan health care system is mainly served by the Family Health Care Workers in various nomenclatures at the grassroots level for the improvement of maternal and child health care status [7]. Family Health Care Workers/ PHMs have a primary role in promotion of vaginal health, not only in prevention through patient education, but also by proper assessment and prompt referrals.
The aim of this study was to develop an assessment form for abnormal vaginal discharge which can be used by Family Health Care Workers/ PHMs, at the community setting and take appropriate actions where necessary. This screening tool will guide the family health care workers to take prompt actions in different cases on an individual basis.

\section{Development of the vaginal discharge assessment form}

The present study focused on developing and validating an assessment form for vaginal discharge. The development of the vaginal discharge assessment form underwent following steps.

a. A review of the literature regarding vaginal discharge.

b. Gathering information on the needs of women's through community survey and focus group discussions.

c. Generation of the vaginal discharge assessment form and judging panel validation.

d. The content and face validation of the developed vaginal discharge assessment form.

\section{A review of literature regarding vaginal discharge}

A preliminary review regarding vaginal discharge and women's health seeking behaviours and cultural practices was conducted in May- June 2014 [8]. Bacterial vaginosis and yeast infections are the most frequently encountered causes of a vaginal discharge but there are numerous other potential aetiologies, both infective and non-infective, that need to be considered in the differential diagnosis [1,2,9,10-12] (Figure 1).

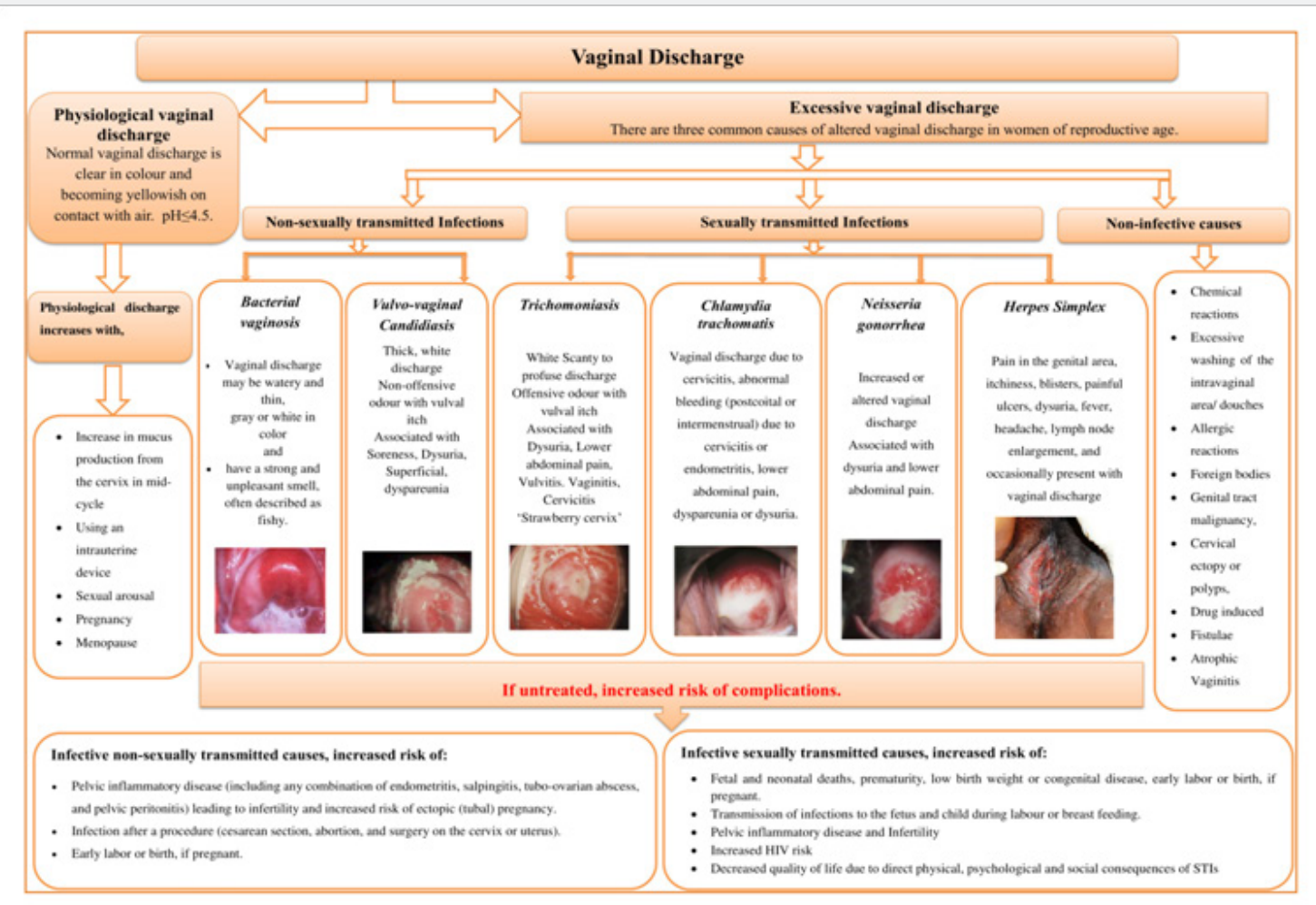

Figure 1: flow chart for the vaginal discharge. 
It was found that embarrassment, anxiety and shame related to disclosing vaginal discharge leads to use of variety of home remedies. Women's lack of knowledge regarding pathological vaginal discharge and use of different cultural practices make them delay seeking medical advice for pathological vaginal discharge [8].

Thus, the literature regarding vagina discharge, its causes and management were identified and ones addressing the question what are the possible causes of abnormal vaginal discharge? were included; studies were selected mainly by virtue of their title and abstract. These literatures were used as a guide for the development of the vaginal discharge assessment form. It was organised in a way that, family health care workers take complete history to disclose the possibility of any of these causes towards abnormal vaginal discharge.

\section{Gathering information on the needs of women's through community survey and focus group discussion}

Descriptive qualitative study was done aiming to assess how women living in estate communities in Colombo District, Sri Lanka manage abnormal vaginal discharge, including their cultural practices and health seeking behaviours [13]. Selfremedies were tried out by the community because of difficulty in disclosing the condition to a doctor, due to perceived stigma and fear of examination and hospitalization and some women mentioned about difficulty in accessing health care, as they are busy with their employment and household work in the present community [13].

Further during a community based survey among 550 women at age of 18 to 49 years it was found that the majority of the study participants had poor knowledge on vaginal discharge displaying the inability to differentiate normal from abnormal vaginal discharge [14].

Through these studies, it was found that women in the low socio-economical setting need additional support and assistance from family health care providers in order to detect pathological vaginal discharge and seek medical advice and treatment.

\section{Generation of the vaginal discharge assessment form and judging panel validation}

Specialists' opinions regarding the content of the vaginal discharge assessment form were obtained by the Delphi technique, which is a widely used and accepted method for gathering data from respondents within their domain of expertise [15]. The technique emerged in the 1950s, created by researchers at the Rand Corporation who sought a method of forecasting the future [16].

Based on the results of the first two phases, the vaginal discharge assessment form was generated and subsequently validated by a panel of judges who were knowledgeable regarding the care of patients with abnormal vaginal discharge. Ten specialists were selected, including five Obstetrician and Gynaecologists, three Consultant Community Physicians, one General Practitioner and one Senior Professor in Community

\section{Medicine.}

Three evaluation rounds using the Delphi technique were proposed. The first was a brainstorming question, "What are the important information should include in history taking of a woman complaining on abnormal vaginal discharge?" The second round included an evaluation of the generated vaginal discharge assessment form based on the information from the integrative review, the focus group, and the results from the first round of discussion. Evaluation in this round focused on the coherence/ pertinence and adequacy/clarity of the information in the vaginal discharge assessment form, as well as the instructions for the actions to be taken for each woman. Finally, in the third round, a final evaluation of the vaginal discharge assessment form occurred after it was amended according to the suggestions made by the specialists during the second round.

The first round included only the open-ended question without a formal instrument. For the rounds that followed, a review form with a three-point Likert scale (Acceptable, Not acceptable, Need changes and Suggestions), based on the items comprising the vaginal discharge assessment form was created. The review forms were assessed and for each statement, agreement above $70 \%$ of the reviewers was taken as it is and below $70 \%$ was discussed and revised. This was assessed qualitatively and the final version of the vaginal discharge assessment form was developed (Supplement 1 \& 2).

\section{The content and face validation of the developed vaginal discharge assessment form}

The vaginal discharge assessment form was developed in English. The developed vaginal discharge assessment form was evaluated for the content and face validity. Face validity is how the variables appear, in order to measure what the investigator wants to measure. Content validity is to check if all aspects of the measures were covered [17].

The experts consisted of Obstetrician and Gynaecologists, Consultant Community Physicians, and Nursing Lecturers. They were invited to check the relevant and suitable guideline for the Sri Lankan setting. They were instructed to read the vulvo-vaginal discharge assessment form carefully and voice any difficulties on testing it and were advised to check for the ambiguity, double barrelled questions and jargon. The experts were requested to give their feedback to the principle investigator. The principle investigator went through the comments of experts for each item.

A $70 \%$ or more total disagreement of any of the items by the experts was considered as invalid and the item was removed from the form. The items with agreement with minor and major changes were highlighted to all the experts. With their approval many of the minor changes were corrected and major changes were changed after majority of the experts agreed to that particular change. The vulvo-vaginal discharge assessment form was modified according to the evaluations made by the expert panel (Figure 2). 


\section{Number.......}

\section{Vaginal Discharge Assessment Form}

1. Sociodemographic information:

a).Name of the patient Miss/Mrs:

b). Age (completed years) .yrs

c). Patient's occupation

d). Husband's occupation.

e). Marital status
a. Single
b. Married
c. Separated
d. Divorced
f). Do you have children?
a. Yes
b. No (go to Q. No 2)
g). Number of children

e. Widow

2. Do you have regular menstruation? a. Yes (go to Q. No 04 )b. No

(go to Q. No 03)

3. If the menstruation has stopped, when did your menstruation stopped?
a. Secondary Amenorrhea
b. Menopause
c. Pregnancy
d. Lactation
e. Contraceptive pills

4. Does your menstrual cycle occur within 21 to 35 days interval?a. Yes

b. No

5. How many days does your menstrual flow last?.

6. Do you have painful menstruation? a. Yes

b. No

7. At present, do you use any contraception or practicing any form of birth control?
a. Yes
b. No
(go to Q. No 09)

8. If yes, what type of contraception you use?
a. Oral contraceptive pills
b. IUCD
c. Implant
d. Depo injection $\square$
e. Condom
f. others
9. Is vaginal discharge bothering you?
a. Yes
b. No

10. How long have you had vaginal discharge?

11. Is it after 8-16 days of menstruation?

a. Yes

b. No

12. Is this the first time you experience excessive vaginal discharge?
a. Yes
(go to Q. No 14)
b. No $\square$ (go to Q. No 13)

13. If "NO", how many times you have experienced it?
a. 2-4 times
b. 5-8 times
c. more than 10 times

14. What is the color of the vaginal discharge?
a. Clear
b. White
c. Yellow
d. Brown

Page 1 of 3 

e. Green
f. others

15. What is the consistency of the vaginal discharge?
a. Thick white
b. Thin
c. Mucoid
d. Frothy

16. What is the odor of the vaginal discharge?
a. non-offensive
b. Offensive
c. Fishy
d. others
(please specify)

17. What other symptoms appear with vaginal discharge?

a. Itchiness and scratching in the genital area $\square$ b. Lower abdominal pain

c. Burning sensation

d. Pain at intercourse

e. Dysuria

g. Fever

i. Vulval edema

f. Post-coital bleeding

f. Abnormal vaginal bleeding

h. Vaginal soreness

e. Intermenstrual bleeding

g. vaginal dryness

h. Others

18. Have you ever taken treatment for excessive vaginal discharge?

a. Yes

b. No

19. Are you currently sexually active? a. Yes (go to Q. No 21)

b. No

20. If no, have you ever been sexually active?

a. Yes

b. No

21. Are you currently taking treatment for any illness?
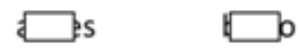

22. Symptoms experience by the husband
a. soreness
b. ulcerations
c. blisters in the penis
d. urethral discharge
e. recurrent dysuria

23. Any other comments?

\section{Actions taken:}

a. Refer the woman to Medical Officer of Health (MOH)/ Gynaecology clinic)

b. Refer the woman to Family Planning Clinic

c. Refer the woman to Well woman clinic

d. Advise to discuss with the relevant physician

e. Advice on personal hygiene and educate woman on normal and abnormal vaginal discharge

Name of the PHM:

Date:

Page $\mathbf{2}$ of 3 


\section{Instructions}

If woman is having vaginal discharge with dysuria, vaginal dryness and she is menopausal (Q. No 03) refer her to well woman clinic.

$>$ If woman is using hormonal contraceptives and having excessive vaginal discharge (Q. No 08), but not associated with other symptoms, refer her to Family Planning Clinic.

> Normal physiological discharge changes with the menstrual cycle. It is thick and sticky for most of the cycle, but becomes clearer, wetter, and stretchy for a short period around the time of ovulation. If the answer for Q.11 is "yes" and colour, consistency, odor are normal and there is no associated symptoms, advice on personal hyqiene.

- Women with persistent or recurrent vaginal discharge (Q. No 13) should be examined and investigated, refer them to $\mathrm{MOH} /$ Gynaecology clinic.

> If woman presents with yellowish, greenish or grayish in colour vaginal discharge, watery or extremely thick, frothy and if offensive, fishy or foul smelling present, (Q. No 14, 15, 16) refer the woman to $\mathrm{MOH} / \mathrm{Gynaecology}$ clinic.

$>$ If woman presents with excessive vaginal discharge with other associated symptoms (Q. No 17), and is she is sexually active, refer them to $\mathrm{MOH} / \mathrm{Gynaecology}$ clinic.

$>$ If woman is taking treatment for any illness and having excessive vaginal discharge (Q. No 21), advise them to discuss with the relevant physician.

$>$ If blood stained or associated with Post coital bleeding refer them to $\mathrm{MOH} / \mathrm{Gynaecology}$ clinic.

Figure 2: Vaginal discharge assessment form.

\section{Translation of vaginal discharge assessment form}

The vaginal discharge assessment form was prepared in English and the first step was to translate the form to Sinhalese and Tamil languages. Translation, back translation method was used during the translation process. The vaginal discharge assessment form was translated to Sinhalese and Tamil language by four independent bilingual (two English - Sinhalese and two English - Tamil) language experts. They were requested by the principle investigator to retain the original structure and content. The principle investigator discussed the variations with the two experts and consensual alterations were made. The agreed Sinhalese and Tamil version of the vaginal discharge assessment form were back translated to English by other English - Sinhalese and English - Tamil language experts. The back translated version was again rechecked with the original vaginal discharge assessment form by the principle investigator for consistency. Any discrepancy was corrected again after discussing with the expert translators.
Practical implications of the developed vaginal discharge assessment form in the community setting

The material design and validation involved scientific knowledge, teamwork, and consideration of the use of the material [16]. The use of the Delphi technique to create the vaginal discharge assessment form, starting with the specialists and including the needs of women in the community, presented a satisfactory outcome. The vaginal discharge assessment form created and validated in this study will assist healthcare personnel to assess women complaining of abnormal vaginal discharge and take appropriate actions at the community level.

From those who had experienced excessive vaginal discharge, only $30 \%$ had taken treatment from a General Practitioner and others have taken other steps or have ignored the symptom in the community based cross sectional study conducted as a preliminary study prior to developing the vaginal discharge assessment form [14]. Further, the majority (73.8\%) have sought medical advice when the condition has got worse and 
most of the participants (87.8\%) stated "feeling uncomfortable in discussing with a male doctor" is the reason for not seeking medical advice for excessive vaginal discharge [14]. Similar results were reported from other studies where women were reluctant to seek treatment because of cultural inhibitions and feeling ashamed in consulting a male doctor [18].

With these evidences, the women living in socially marginalised communities are highly vulnerable for the associated consequences of untreated pathological vaginal discharge. The majority of these women would communicate their health matters especially reproductive health matters, with the family health care workers who have close relationship with these communities.

In Sri Lanka the community-based health care is delivered through health units managed by the Medical Officer of Health $(\mathrm{MOH})$ and within each health unit, home visits made by the public health midwife (PHM) [19]. The PHM is a specially trained front-line family health care provider for the mother and child, providing both home and clinic based care. The PHM training period is for 18 months with 1 year in a nurse's training school and the rest in a field training centre [20-22]. PHMs conduct field visits in order to ensure the health and well- being of their allocated population [21]. During her home visits for antenatal -postnatal care and child care services, she meets women in reproductive age. As all PHMs are female, they can build trusting relationships with women in the community [21]. This will create an encouraging environment for them to discuss their reproductive and sexual health matters. Further, during their antenatal and postnatal examinations, they would come up with pregnant women having abnormal vaginal discharge.

A survey done to identify essential public health functions of field health staff in Sri Lanka, indicated that PHMs accept that health promotion is a function applicable to their work [23]. However, the most stated perceived benefit of health promotion approach was that it makes the duties of a PHM easier which is contrary to the common belief that it increases the work load of PHMs, adding more burden to them [23]. Therefore the introduction of a tool to assess vaginal discharge will not be an additional burden to them, but will make their life easier.

As the most available family health care worker, PHM has a great role in promotion of vaginal health of women, especially those living in socially marginalised communities. The guidelines or tools of screening a disease condition will help the family health care workers who are not specialised in a field (where their specialisation differ) to take appropriate actions. Some other assessment tools such as premenstrual symptoms screening tool has been identified as user-friendly, and has been found to aid Family Practitioners, Obstetricians, Gynecologists, Paediatricians, Psychiatrists, and other healthcare professionals in assessing adolescent girls for premenstrual mood disorders [24]. Further, the home safety assessment tool is another example of an assessment protocol which can used to identify environmental barriers that can be modified or removed to provide a safe and functional environment for clients [25]. Furthermore, another such screening tool is Postpartum Depression Predictors Inventory (PDPI), a checklist designed to identify women at risk for developing postpartum depression which can be used to continue monitoring the mother's risk status [26].

Although there is no such evidence of developing vaginal discharge assessment tool in the literature, this is a preliminary effort to help the family health care workers to be confident in assessing and taking complete history regarding vaginal discharge from a woman in order to decide the future management. The instructions at the end of the form, give an idea about the accurate measure the health care provider should take. Further, the documentation taken as an important practice for the health care worker will further help in her professional advancement.

The vaginal discharge assessment tool will further improve the health seeking for abnormal vaginal discharge among the community as the family health care workers are well organised and prepared to assist needy women and reduce the unnecessary consultations and health care cost. Additionally, the developed vaginal discharge assessment form will be a guide for family health care workers who are mainly engaged in maternal and child care services and will reduce the stress associated with addressing other health maters among community and it will minimize the errors of faulty diagnosis and inappropriate decisions on vaginal discharge management at community level.

\section{Recommendations to practice}

Vaginal discharge is a topic silent in nature as most women are not aware of the change of the normal from abnormal. In women from lay population, where the health literacy is low and socio cultural background is poor, the health seeking patterns for vaginal discharge and disclosure of the vaginal health matters are poor. Another important aspect of this population is their risk behaviours which make them more vulnerable for sexually transmitted infections and other reproductive tract infections. Family health care workers who attend for the health promotion of the women in rural and urban slum communities have a great role in identifying risk women and assessing them for any reproductive pathologies. The developed vaginal discharge assessment form guide could assist the family health care workers to assess a woman for the required details for deciding the accurate actions to be taken.

Family health care worker can decide to refer the woman to the family planning clinic, well- woman clinic, gynaecological clinic when a pathological vaginal discharge is suspected. Further, when identified as physiological excess vaginal discharge, family health care provider can advise the woman on vaginal health, personal hygiene, physiological reasons for excessive vaginal discharge, preventive behaviours for reproductive tract infections and condom use for prevention of sexually transmitted 
infections. Therefore the vaginal discharge assessment form is a simple tool which can assist the daily practice of a family health care worker to promote women's health.

\section{Author's' contributions}

Conception and design of study: IMPS, CSE, PPR.

\section{Acquisition of data: IMPS}

Analysis and/or interpretation of data: IMPS, CSE, PPR, RC.

Drafting the manuscript: IMPS

Revising the manuscript critically for important intellectual content: IMPS, CSE, PPR, RC.

Approval of the version of the manuscript to be published: IMPS, CSE, PPR, RC.

\section{Ethical Approval}

Ethical clearance was obtained from the Ethics Review Committee, Faculty of Medical Sciences, University of Sri Jayewardenepura, Sri Lanka. Recruitment of all participants for the study was strictly voluntarily.

\section{References}

1. Henn E, Kruger T (2005) Vaginal discharge reviewed : the adult premenopausal female. SA Fam Pract 47(2): 30-38.

2. Mashburn J (2012) Vaginal Infections Update. J Midwifery Womens Health 57(6): 629-634.

3. Venkataram Y, Al-Suwaid R (1997) Syndromic management of sexually transmitted diseases. Genitourinary medicine 73(4): 324.

4. WHO (1004) Management of Sexually Transmitted Diseases. World Health Organization, Geneva, Switzerland.

5. Niriella NC (2010) Critical Issues of Low-Income Dwellers in Urban Planning. pp. 1-15.

6. Annual Report (2016) National STD/AIDS Control Programme. Ministry of Health, Sri Lanka.

7. Ilankoon IMPS, Goonewardena CSE, Fernandopulle RC, Perera PPR (2016) Public Health Midwives' role in health education regarding vaginal discharge: A Cross Sectional Descriptive Study. International Journal of scientific research and management 4(6): 4303-4310.

8. Ilankoon IMPS, Goonewardena CSE, Fernandopulle RC, Perera PPR (2015) Research Article Vaginal Discharge : Women's Health Seeking Behaviours and Cultural Practices. International Journal of Current Research 7(6): 17473-17479.

9. Andrist LC (2001) Vaginal Health and Infections. Journal of Obstetric, Gynecologic and Neonatal Nursing 30: 306-315.

10. Bates S (2003) Vaginal discharge. Current Obstetrics \& Gynaecology Instructions:
13: $218-223$.

11. Fahami R (2013) Abnormal vaginal discharge. BMJ 347: f4975.

12. McCathie R (2006) Vaginal discharge: common causes and management. Current Obstetrics \& Gynaecology 16(4): 211-217.

13. Ilankoon IMPS, Goonewardena CSE, Fernandopulle RC, Perera PPR (2017) An Insight to How Estate Women in Sri Lanka Manage Abnormal Vaginal Discharge. Iran J Public Health 46(2): 268-269.

14. Ilankoon IMPS, Goonewardena CSE, Fernandopulle RC, Perera PPR (2017) Women's Knowledge and Experience of Abnormal Vaginal Discharge Living in Estates in Colombo District, Sri Lanka. International Journal of Women's Health and Reproduction Sciences 5(2): 90-96.

15. Linstone H (1978) The Delphi technique. Handbook of futures research $12(10)$.

16. Sousa CS, Turrini RNT (2012) Creating and validating educational material for patients undergoing orthognathic surgery. Asian Nursing Research 6(4): 166-172.

17. Abramson JH, Abramson ZH (2008) Research Methods in Community Medicine; Surveys, Epidemiological Research, Programme Evaluation, Clinical Trails. (6 $6^{\text {th }}$ edn), John Wiley \& Sons Ltd, USA.

18. Malta M, Bastos F, Strathdee S, Cunnigham S, Pilotto J, et al. (2007) Knowledge, perceived stigma, and care-seeking experiences for sexually transmitted infections: a qualitative study from the perspective of public clinic attendees in Rio de Janeiro, Brazil. BMC Public Health 7: 18.

19. Sivaganesh S, Senarath U (2009) Antenatal Care Utilization in a Conflict-Affected District of Northern Sri Lanka. Public Health Nursing 26(6): 512-522.

20. Senanayake H, Goonewardene M, Ranatunga A, Hattotuwa R, Amarasekera S, et al. (2011) Achieving Millennium Development Goals 4 and 5 in Sri Lanka. BJOG 118(Suppl 2): 78-87.

21. Jayatilleke AC, Yoshikawa K, Yasuoka J, Poudel KC, Fernando N (2015) Training Sri Lankan public health midwives on intimate partner violence : a pre- and post-intervention study. BMC Public Health 15: 331.

22. Infanti JJ, Lund R, Muzrif MM, Schei B, Wijewardena K (2015) Addressing domestic violence through antenatal care in Sri Lanka's plantation estates: Contributions of public health midwives. Soc Sci Med 145: 35-43.

23. MN Perera, Guruge ND, Gunawardena NS (2015) Knowledge on Health Promotion among Public Health Midwives in a District in Sri Lanka. Journal of the Postgraduate Institute of Medicine 2: 1-10.

24. Steiner M, Peer M, Palova E, Freeman EW, MacDougall M, et al. (2011) The premenstrual symptoms screening tool revised for adolescents (PSST-A): Prevalence of severe PMS and premenstrual dysphoric disorder in adolescents. Archives Arch Womens Ment Health 14(1): 77-81.

25. Romero S, Lee MJ, Simic I, Levy C, Sanford J (2017) Development and validation of a remote home safety protocol. Disability and Rehabilitation: Assistive Technology 13(2): 166-172.

Please individually rate following 36 items acceptable or not acceptable. Please fill in your comments/ rating in the space following each question number (vulvo-vaginal discharge assessment form and the review form attach herewith). You may also provide additional comments that identify deficient areas and provide recommendations, suggestions on ways to improve the vulvo-vaginal discharge assessment form.

Please send you comments form to prasanthiilankoon@yahoo.com e mail address.

Thank you for your support.

Yours Sincerely, 


\section{Journal of Gynecology and Women's Health}

\section{Ms.I.M.P.S.Ilankoon}

Supplementary File 2: Vulvo- vaginal discharge assessment form: Review Form.

Please indicate $(x)$ mark in the appropriate box and indicate your suggestions in given space.

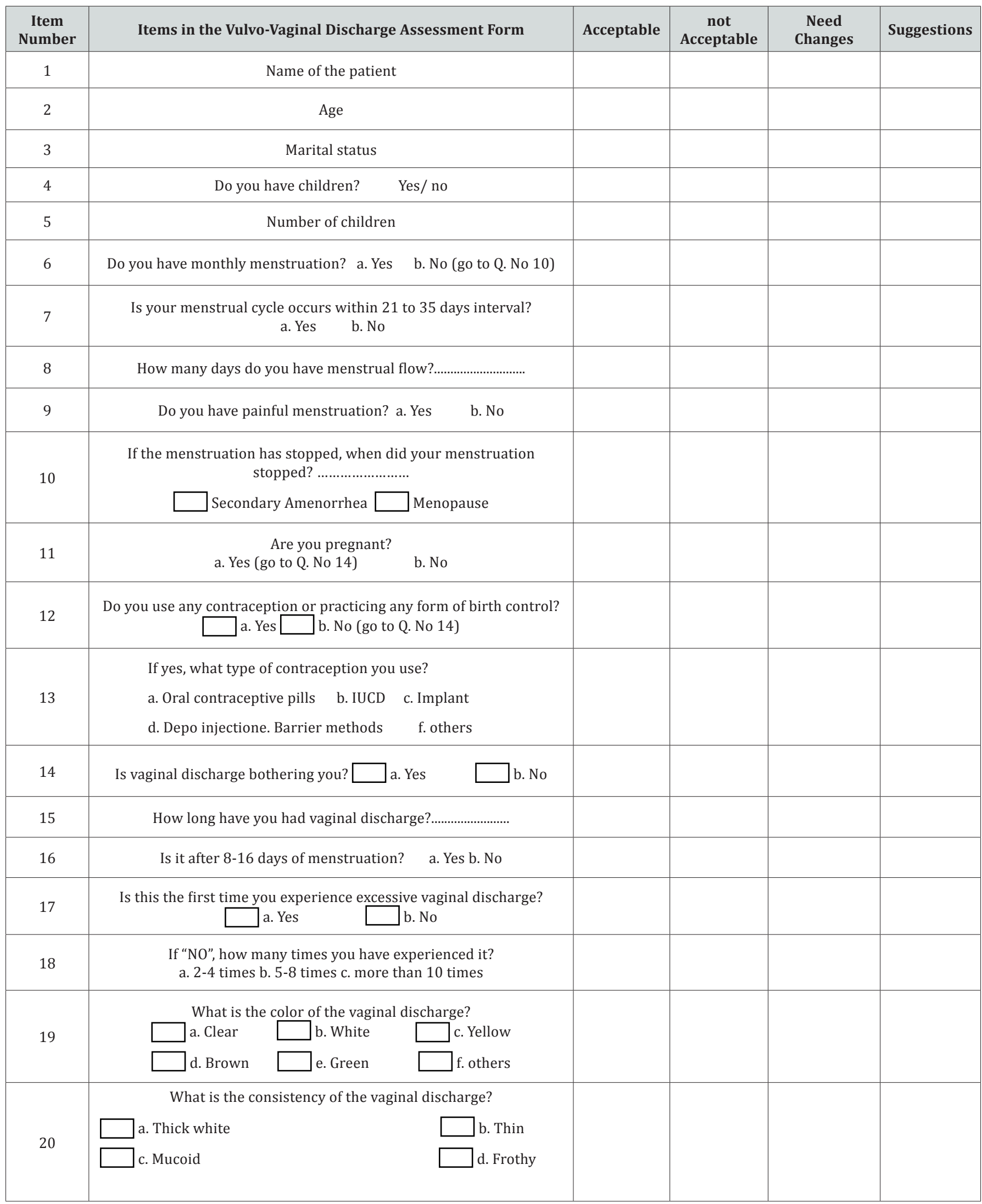




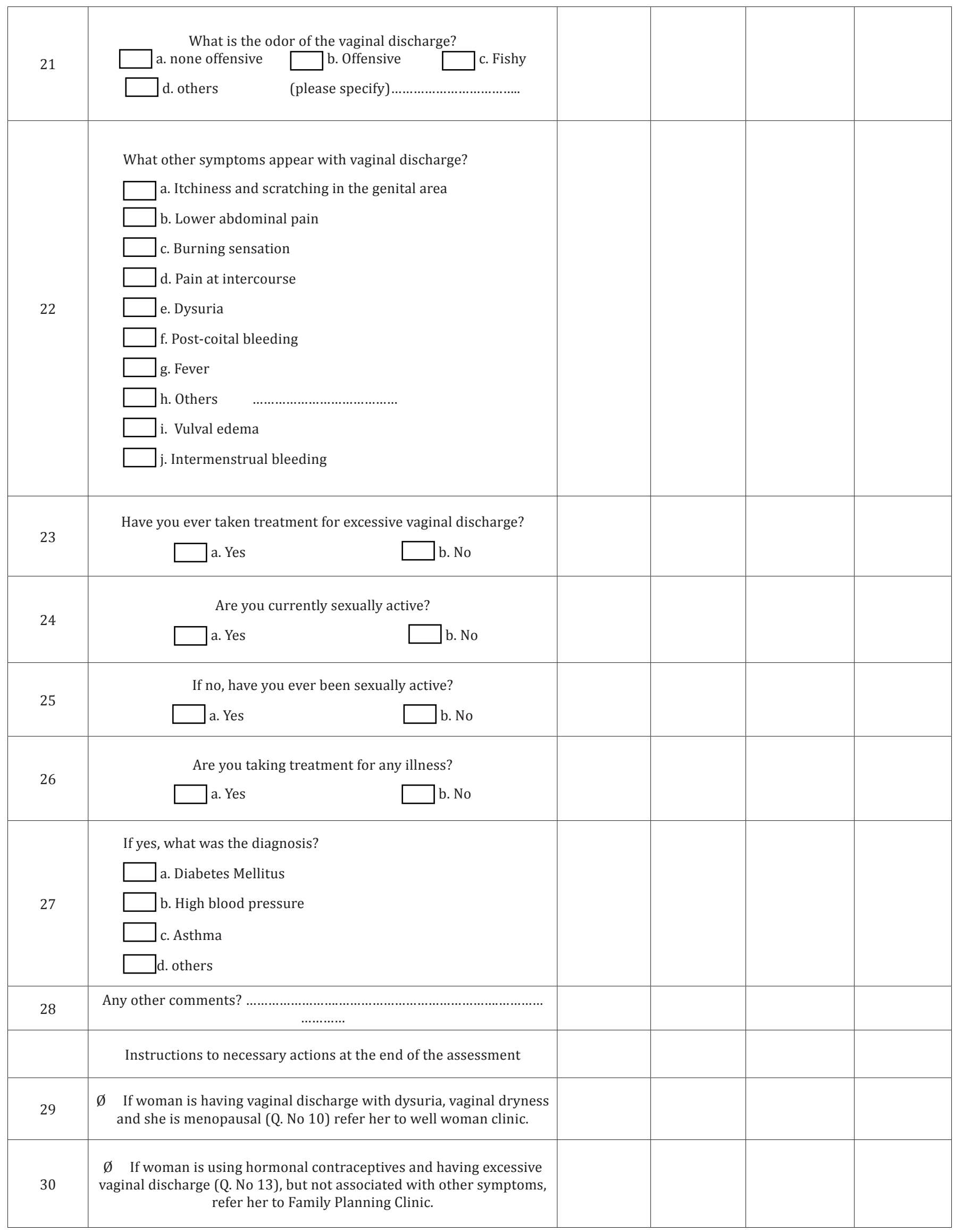




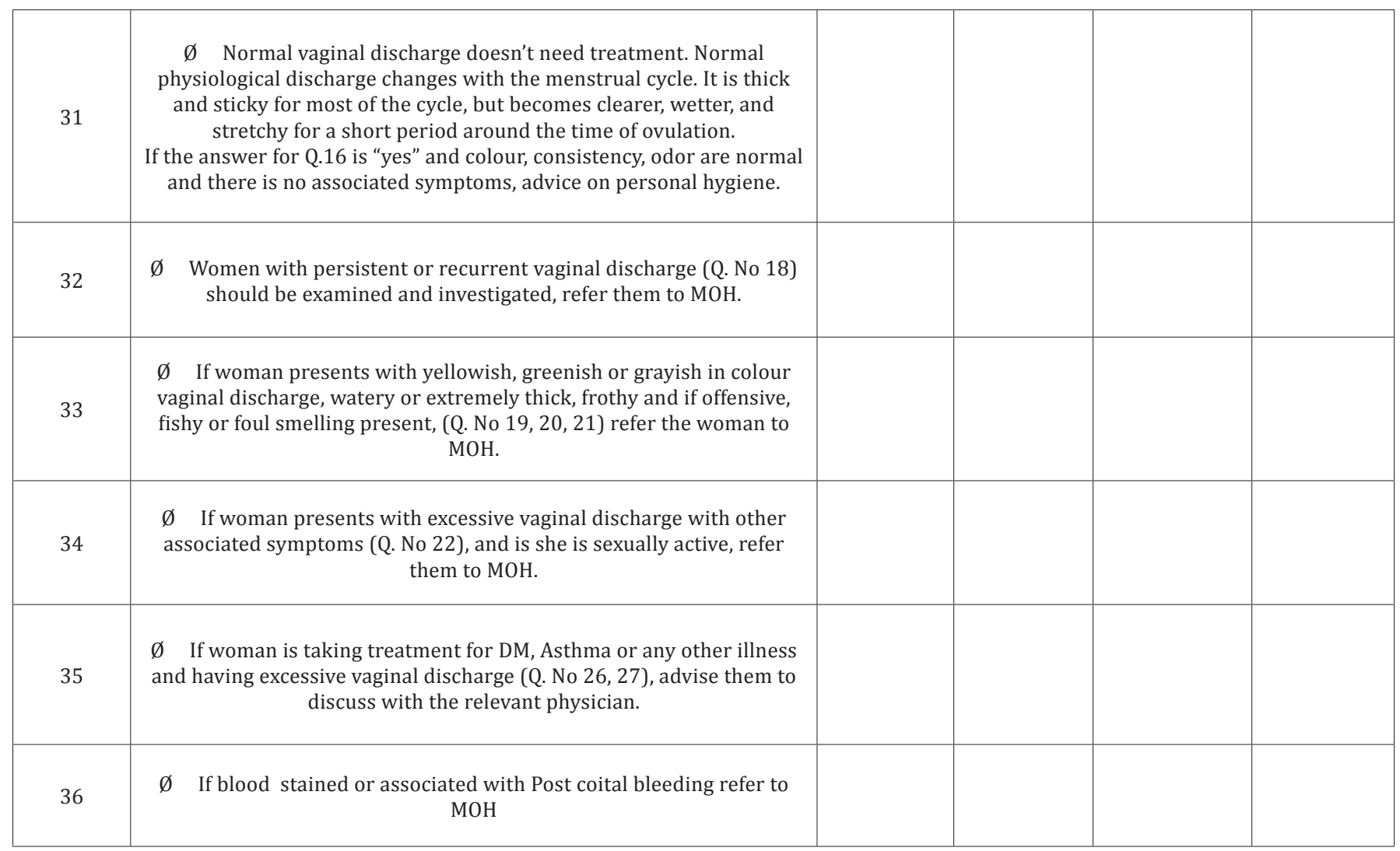

Please indicate any suggestions/ comments necessary for improve this Vulvo- vaginal discharge assessment form:

Name of the reviewer:

Signature:

Date:.

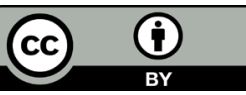

This work is licensed under Creative Commons Attribution 4.0 License DOI: 10.19080/JGWH.2018.09.555763

\section{Your next submission with Juniper Publishers} will reach you the below assets

- Quality Editorial service

- Swift Peer Review

- Reprints availability

- E-prints Service

- Manuscript Podcast for convenient understanding

- Global attainment for your research

- Manuscript accessibility in different formats

( Pdf, E-pub, Full Text, Audio)

- Unceasing customer service

Track the below URL for one-step submission https://juniperpublishers.com/online-submission.php 